\title{
Treatment of malocclusions in the temporal period of bite, children with speech disorders by means of myogymnastics and face tapping.
}

\section{LYKHOTA Kostiantyn, PETRYCHENKO Oleksandra, ARDYKUTSE Vasylyna, MYKHAILOVSKA Larysa, KUTSIUK Anatoii}

Corresponding author: LYKHOTA Kostiantyn, E-mail: k.lykhota@lykhota.kiev.ua

Abstract

Shupyk National Medical Academy of Postgraduate education, Kyiv, Ukraine

Introduction: The article deals with the current problems of modern orthodontics, aimed at increasing the effectiveness of treatment of sagittal anomalies of bite, which are complicated by speech disorders, patients in the period of bite. The effectiveness of the proposed methods of treatment is investigated, and their complex approach to the correction of this pathology is evaluated.

Materials and methods: We were taken on the treatment of 47 children with temporary bite, aged 3 to 5 years old, who experienced dental anomalies in combination with organic dyslallia. Depending on the pathology of bite, patients were divided into subgroups A and B. Subgroup A included children with distal bite, to subgroup B - with mesial.

According to the chosen method of orthodontic treatment, patients of experimental groups were additionally divided into 2 subgroups. In 1 subgroup included children, the treatment of which was carried out according to the proposed method, to 2 subgroups - according to the standard.

The control group included 11 children with physiological bites of similar age without speech disorders.

Results: The effectiveness of proposed schemes of orthodontic and logopedic rehabilitation was proved by clinical, anthropometric and functional indicators, which were determined prior to and at different times after starting treatment. Clinical efficacy of performed orthodontic treatment is established on the term of treatment, normalization of articulation motility, normalization of anthropometric indices, normalization of palatogram characteristics, indexes of functional activity of the tooth-jaw system, normalization of occlusal ratios of dentitions.

Conclusion: Using the proposed method of orthodontic treatment (with the use of orthodontic equipment in combination with modified myogymnastics and facial tapping), the dynamics of all the studied parameters correlated with the timing of the pathology elimination and indicated an increased effectiveness of treatment in applying the proposed method, namely: all patients had normalization forms of dental arches, normalized indexes of width and length of the dental arc. Immediately after the end of the treatment, electromyographic indices were normalized and occlusive relationships restored. Similar indicators for those who were treated according to the standard method, although they showed positive dynamics, but differed both from the indicators of persons who were treated according to the proposed method, and from the indicators of the control group.

Key words: maxillofacial area, malocclusions, sagittal anomalies, speech disorders, tapping,

\section{Introduction}

The questions of etiology, clinic and pathogenesis of anomalies and deformations of the maxillofacial area become especially relevant as their prevalence is constantly increasing. Despite the rapid development of orthodontics, the quality of treatment for children with of malocclusions, complicated speech disorders remain unresolved (14).

One of the most common causes of the wrong sound is the incorrect structure of the articulatory apparat. At the same time, disturbance of sound and improper articulation, infantile type of swallowing, violation of the tone of chewing and mimic muscles cause anomalies and deformation of the dental-jaw area (57).
In recent years, the study of the relationship and interdependence of malocclusion and articulatory disorders has been conducted. New methods of research of patients are being developed, possibilities of cooperation of orthodontist dentistry with specialists of other medical branches are expanded (8-10).

In this study the method of treatment of malocclusions, children with speech disorders was substantiated by the complex application of vestibular plates, modified myogymnastics and face tapping.

This made it possible to achieve complete normalization of all clinical and functional parameters of orthodontic treatment in much shorter time than patients treated with the commonly accepted method. 
The features of clinical and functional parameters in patients with sagittal bite anomalies, which are complicated by organic dyslalia, before and after treatment, are investigated in the work, which makes it possible to comprehensively approach this problem.

The comparative estimation of efficiency of use of the offered method of treatment of sagittal anomalies complicated by speech disorders, children in a temporary period of bite with the generally accepted methods is carried out.

\section{Aims}

Improvement of the effectiveness of treatment of sagittal dento-jaw anomalies, children with a disturbance of the pronunciation of sounds during a temporary bite by the use of orthodontic equipment, modified myogymnastics and face tapping.

\section{Materials and methods}

The first stage of work was to determine the morpho-functional parameters of the dental-jaw system, children with speech disorders. Depending on the pathology of bite, 47 patients aged 3 to 5 with a temporary bite, which had dental anomalies in combination with dyslalia, were divided into two main groups: A and B. Group A included children with distal occlusion, in the group B - with mesial.

The second stage of the work was the orthodontic treatment of patients with speech disorders. According to the chosen method of orthodontic treatment, patients of experimental groups were additionally divided into 2 subgroups. In one subgroup of the two main groups included children, the treatment of which was carried out according to the proposed method, to 2 subgroups - according to the standard. The control group consisted of 11 children with physiological bites of similar age without speech disorders, which served as control of the effectiveness of the conducted orthodontic treatment.

The treatment of children with both distal (group A) and mesial (group B) bites was carried out according to the proposed method (vestibular plates with a visor (the direction of the visor depended on the type of bite) in combination with modified myogymnastics and tapping) and the standard method (vestibular plates with standard mogimnastics).

To increase the effectiveness of the treatment the combined use of detachable orthodontic equipment was proposed, myo-gymnastics and face taping.
Myo-gymnastics was aimed at activating spontaneous facial expressions and the formation of arbitrary facial muscle movements. Also there was a set of exercises used to normalize the tone of the circular muscle of the mouth and a set of exercises to normalize the tone of the muscles of the tongue. In addition, a complex of modified myo-gymnastic exercises was used. Here is a set of exercises to normalize the tone of the tongue: "Football": put a round candy on a plate, form a "gate" of two fingers; the child must push the "ball" into the "gate" with the tongue; "Kolobok": a round candy is located on the tongue of the child like on a plate; "Wintersummer": a spoon in turn is immersed in cold water, then in warm, it is necessary to place a spoon on the side parts of the tongue. A set of exercises for the normalization of the tone of the circular muscle: "Somyk (Catfish)": Lips are closed and protruding, an "elephant" exercise, sweet straw is placed between the upper lip and the nose, which should be kept for as long as possible.

Myo-gymnastic exercises were prescribed for a period of 5-6 months.

Kinesiological taping helps normalize the function of circular muscle of the mouth, functions and position of the tongue, normalize the opening of the mouth and the process of swallowing also stabilizes the position of the lower jaw. When applying tape, the microcirculation and lymph flow in the adjacent tissues are improved, as well as the irritation of the proprioceptors occurs in the area where the tape is bonded, forcing the brain to pay attention to muscles that function incorrectly. Tape is fixed for a period of 1 to 4-5 days.

Pathology of bite was evaluated according to the Angle classification (1898).

Diagnosis of "mechanical dyslalia" was established by the speech therapist-defectologist.

Measurements of transversal and sagittal size of the jaws were performed by the Dolgopolov method; in the second group, the measurement of the width of the dental arches was carried out using the Pont method (as amended by H. Linder, G. Hart) and Korkhaus. Also, forms of the dental arches of the upper and lower jaw were determined.Functional research methods included:

- palatography by methodology Smagljuk L. V. and Trofimenko M. V. (2008); - examination of articulation motility;

- the state of speech: sound and speech of the breath; 
- Electromyographic study of the activity of chewing muscles with the help of the computer-aided neuroelechemographer M-Test of the association of the DX system (Kharkiv) with the definition of quantitative and qualitative indices of bioelectric activity of chewing muscles;

- Occlusion study by computerized analysis of TScan III (USA) occlusion with the definition of quantitative and qualitative indicators.

Informed voluntary consent to the processing of personal data was signed with the parents of all patients.

The results of clinical and laboratory studies were processed by the method of variation statistics using the computer programs Microsoft Excel 2010 and Origin Pro 7.5.

The reliability of the detected differences was evaluated according to Student's criterion. The differences were considered significant at $p<0.05$.

\section{Results}

The effectiveness of the proposed schemes of orthodontic and logopedic rehabilitation was proven by clinical, anthropometric and functional indicators, which were determined prior to and at different times after starting treatment. Clinical efficacy of performed orthodontic treatment is established on the term of treatment, normalization of articulation motility, normalization of anthropometric indices, normalization of palatogram characteristics, indexes of functional activity of the dental-jaw system, normalization of occlusal ratios of dentitions.

The results showed the effectiveness of the treatment, which resulted in the restoration of both the normalization of all functions of the maxillofacial area and articulation motility.

The terms of treatment for most $(66 \%)$ patients ranged from 6 to 12 months. Less than 6 months of treatment lasted only in $12,8 \%$ of patients, and more than 12 months - in $21,2 \%$.

The terms for eliminating bite anomalies and normalizing articulation motility in the application of the proposed treatment method in the vast majority of children (72.8\%) ranged from 6 to 12 months. At the same time, it should be noted that in the period up to 6 months, the elimination of pathology was found in a significant $(22.7 \%)$ number of patients. Only one person $(4.5 \%)$ with distal occlusion needed orthodontic treatment for more than 12 months.

The duration of treatment in children with distal and mesial bite was approximately the same and ranged from an average of 6 to 12 months. During the specified observation period, along with elimination of occlusion anomalies and normalization of articulation motility, elimination of bad habits took place (Fig. 1-5). Already after 3 months, the use of orthodontic technique in conjunction with the modified myogymnastics and tapping, we observed the elimination of bad habits of sucking fingers and foreign objects, tucking and sucking the tongue. It took an average of 6-7 months to eliminate the problem of swallowing, mouth breathing and speech impairment, while at the same time the observation was normalized posture and position of the mandible and tongue at rest. In parallel, pathology of soft tissues was eliminated, namely, a violation of the tone of the circular muscle and tongue (Table 1).

\section{Table 1 Terms of treatment for patients}

\begin{tabular}{|c|c|c|c|c|c|c|c|c|}
\hline \multirow{3}{*}{ № } & \multirow[t]{3}{*}{ № } & \multirow{3}{*}{$\begin{array}{l}\text { Lines } \\
\text { elimination of } \\
\text { pathology } \\
\text { (months) }\end{array}$} & \multicolumn{6}{|c|}{ Results of treatment } \\
\hline & & & \multicolumn{2}{|c|}{ A (distal bite) } & \multicolumn{2}{|c|}{ B (mesial bite) } & \multicolumn{2}{|c|}{ Total } \\
\hline & & & абс. & $\%$ & абс. & $\%$ & абс. & $\%$ \\
\hline \multirow[t]{3}{*}{1 subgroup } & \multirow[t]{3}{*}{22} & $<6$ & 2 & 4,3 & 3 & 6,4 & 5 & 10,7 \\
\hline & & $6-12$ & 9 & 19,1 & 7 & 14,9 & 16 & 34,0 \\
\hline & & $12 \mathrm{i}>$ & 1 & 2,1 & - & - & 1 & 2,1 \\
\hline \multirow[t]{4}{*}{2 subgroup } & \multirow[t]{3}{*}{25} & $<6$ & - & - & 1 & 2,1 & 1 & 2,1 \\
\hline & & $6-12$ & 8 & 17,0 & 7 & 14,9 & 15 & 32,0 \\
\hline & & $12 \mathrm{i}>$ & 5 & 10,7 & 4 & 8,5 & 9 & 19,1 \\
\hline & 47 & & 25 & 53,2 & 22 & 46,8 & 47 & 100 \\
\hline
\end{tabular}



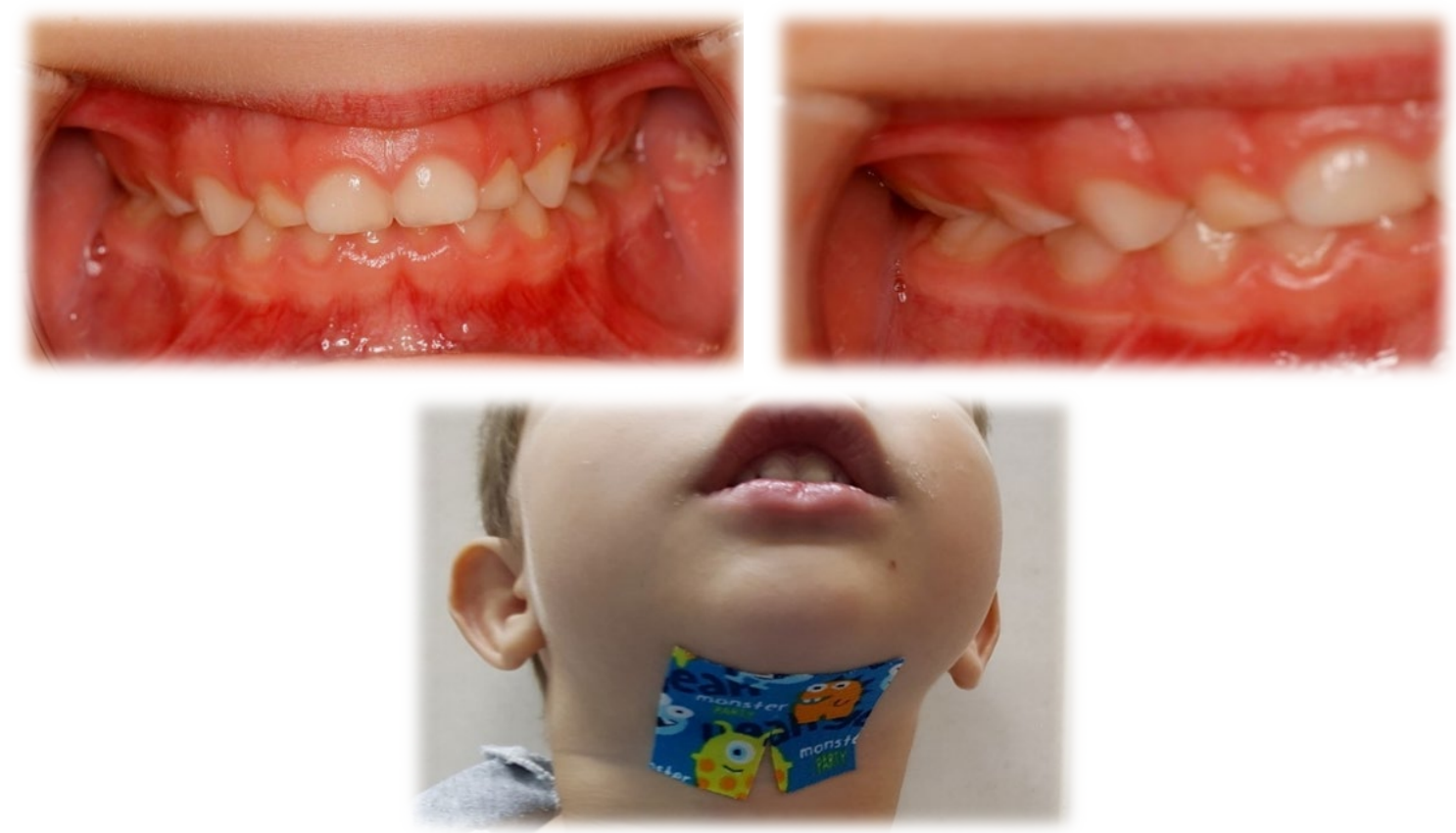

Fig. 1-3 Patient's D. photo 4 y. o. before treatment
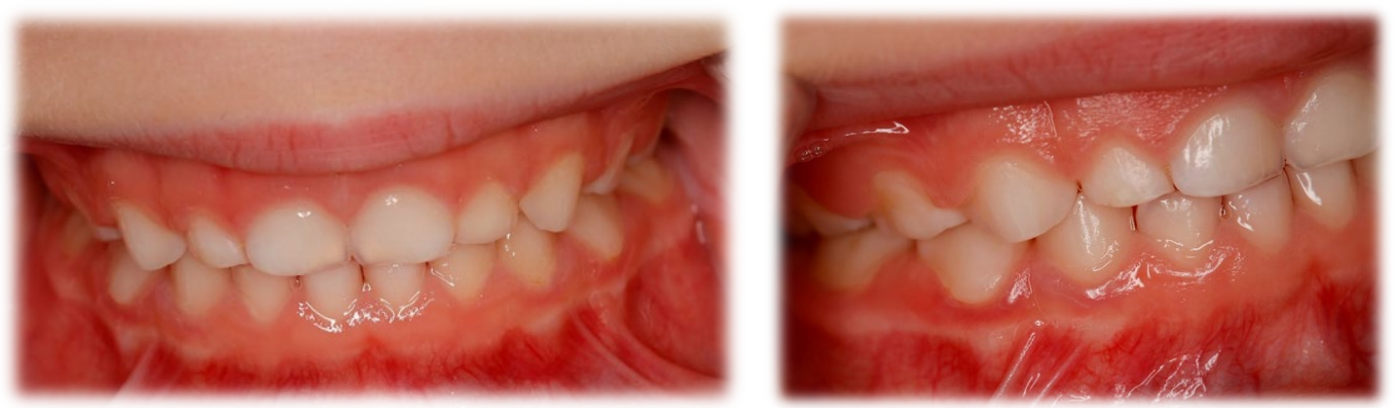

Fig. 4, 5 Patient's D. photo 5 y. o. after treatment

The form of dental arches has been normalized in all patients using both the proposed and standard treatment methods.

Normal values of width and length of the dental artery in 12 months were diagnosed according to the 1 st and 2nd subgroups at $95.4 \%$ and $84.0 \%$ respectively. After the treatment, the normalization of palatogram rates for pronunciation and swallowing in the A1 subgroup was observed in $91.7 \%$ of subjects, and in A2 - 84.6\%. In subgroup B1 at $100 \%$, and B2 in $83.3 \%$. After completion of treatment, articulation motility met the norm in subgroup A1 in $91.7 \%$ of subjects, in $\mathrm{A} 2$ - at $76.9 \%$, in $\mathrm{B} 1$ - at $100 \%$, in $\mathrm{B} 2-75.0 \%$. And indicators of speech research in subgroup A1 and B1 in $100 \%$ of people, in A2 - 84.6\%, in B2 - 91.7\%.

Speech rates in subgroup A1 normalized in $88.9 \%$ of the people, in $\mathrm{A} 2-70.0 \%$, in $\mathrm{B} 1-90.9 \%$, in $\mathrm{B} 2-$ $80.0 \%$.
The functional activity of the dental-jaw system in patients with the application of the proposed method of orthodontic treatment was restored immediately after treatment, the mean compression amplitude was $546 \pm 5.9 \mathrm{MkV}$ and $489 \pm 7.5 \mathrm{MkV}$ according to the right and left chewing muscle, and practically corresponded to the control groups, in the 2nd subgroup, similar indices were $477 \pm 4.7 \mathrm{MkV}$ and $418 \pm 8.4 \mathrm{MkV}$, respectively.

The occlusion study revealed that the normalization of occlusive violations in patients occurred in parallel with the normalization of functional activity of chewing muscle in all patients who had orthodontic treatment. However, both qualitative and quantitative indexes of occlusion in patients who used the proposed method of orthodontic treatment were much faster than normal. 
If before the beginning of orthodontic treatment in patients, the index of asymmetry of relative strength between the sides of the dental rows increased significantly and led to a non-physiological redistribution of loads compared with the control group due to the inconsistency of occlusal contacts and was $-14.7 \pm 3.9 \%, 13.2 \pm 4,2 \%$ and against similar control indicators $-5.1 \pm 1.2 \%$ respectively $(p \leq 0.05)$. In all patients, an increase in the time of onset of the maximum number of dental contacts $(0.42 \pm 0.02 \mathrm{~s})$ was found for persons with distal occlusion compared with the control parameters $(0.27 \pm 0.02 \mathrm{~s}) \mathrm{p} \leq 0.05$.

After 12 months of treatment, almost all patients who used the proposed procedure disappeared premature occlusal contacts, the direction of the trajectory of the total vector of occlusion was normalized against the backdrop of an increase in the period of bioelectric activity and a decrease in the rest period and the normalization of the coefficients $\mathrm{K}$. Thus, after 12 months of treatment with standard orthodontic the occlusion time of the appliance was $0.25 \pm 0.01 \mathrm{~s}$ and $0.225 \pm 0.03 \mathrm{~s}$ in accordance with the distal and mesial bites, whereas the same indices in subgroups A1 and B1 amounted to $0.21 \pm 0.03 \mathrm{~s}$ and $0.20 \pm 0.01 \mathrm{~s}$ respectively). Similar patterns were found during the study of the appearance of the maximum number of dental contacts and the time of disocclusion.

The developed treatment methods allow not only to shorten the treatment period, but also to normalize the impaired functions of the dental-jaw system and to eliminate harmful habits, patients with this pathology.

\section{Discussion}

Dyslalia is a speech impairment in normal hearing and preserved innervation of the speech appliance. Distinguish between mechanical and functional dyslalia. Mechanical (or organic) dyslalia is a consequence of organic defects of the peripheral speech appliance, that is, articulation organs. The organical dyslalia leads to a shortened bridle of the tongue, a change in the size of the tongue, tooth-jaw anomalies and deformations, a change in the structure of the palate, and a violation of the function of the lips (4).

The violation of articulation is one of the important local factors in the occurrence of malocclusions $\mathrm{Yu}$ L. Obraztsov believes that this factor is the most important in the etiology of mesial bite and it remains significant in the formation of most malocclusions, with the exception of deep bite (11, 12).

Y. M. Maligin points out that improper pronunciation is often a sign of disturbance of the central nervous system, as well as the result of improper development of firm and soft tissues of the oral cavity. The truncated bridle of the tongue and its irregular articulation lead to the deformation of the dental alveolar arches, in particular to the open bite.

Such anomalies of the structure of the cavity of the mouth as the shape of the palate, the position of the teeth, the shape and position of the tongue in rest, and other functions of the dental-jaw system $(13,14)$ play a special role in clarity of pronunciation of sounds.

The interrelation and interdependence of malocclusions and articulatory disorders were emphasized by V. D. Kuroyedova and her coauthors (4) in his studies, noting that the anomalies of the appliance of articulation sharply distort the pronunciation and speech-work without orthodontic correction turns out to be ineffective. According to the same authors, only $24.3 \%$ of the subjects with malocclusions correspond to the generally accepted phonetic norms, but the articulation of the tongue is incorrect, which represents the greatest difficulty in diagnosing for a practical orthodontist [9].

Violations of the sound may be caused by two main groups of factors: anatomical deviations in the structure of the articulation appliance and violations of innervation of the various parts of the appliance of articulation (central or peripheral genesis).

T. Y. Nemish concludes that constant logopedic training does not positively influence the processes of self-regulation of tooth-jaw anomalies in severe forms of speech disorders; therefore, it recommends orthodontic treatment in such children as early as possible.

V. D. Kuroyedova and co-authors (4) indicate that the shape of the palate, the position of the teeth, and especially the tongue, greatly affect the quality of pronunciation.

Tongue is the most important organ of articulation. According to the same authors, articulation is violated as a result of changes in the shape and area of the palate, the closure of the lips, the location of the tongue between the dental rows. Also, the mobility of the tongue is disturbed when the 
anomalies of the development of soft tissues, in particular the anomalies of the tongue bridles. However, the volume of the tongue, according to Y. O. Gioeva and co-authors, has little effect on the size of the jaws and the inclination of the jaws of the upper jaw (7).

L. Volkov and colleagues assert that in all cases of mechanical dyslalia, consultation and treatment from such specialists as surgeon and orthodontist is necessary. The earlier the bite pathology appeared, the greater the risk of occurrence of persistent sound defects. Malocclusions in a temporary occlusion provoke more stable violations of the sound side of the tongue than those that manifested themselves only in a constant bite $(11,12)$. The earlier the pathology of bite, pathology of tongue, lips were revealed and the earlier work on elimination of defects of articulation organs, violations of sonority and the development of phonemic perception was started, the faster and more efficiently we can achieve positive results in the correction of the organic dyslalia.

L. Y. Yevtushenko and his co-authors found that the effect of the existing irregular articulation of the tongue on the anterior teeth in patients with III class by Engle

was characterized by swallowing efforts that far exceed the chewing effort. The results of the removal of the tongue from the anterior teeth indicate not only the participation in the formation of pathology, but also on the polyethiologicity of the latter (13).

Similar results were received by other researchers, confirming the effect of irregular articulation of the tongue on the formation of buccal pathology, and also proving that the efforts on the anterior teeth with irregular articulation are higher than normal. The pressure when swallowing on the front teeth significantly exceeds the force of chewing and sounding $(4,6)$.

Consequently, malocclusions have a polyethiological nature. Violation of pronunciation, its articulation side, can be considered as one of the important causative factors of bite pathology, which is least covered in modern scientific orthodontic literature.

Thus, the earlier the correction of speech disorders begins, the higher its effectiveness. An integrated approach plays a significant role in the rehabilitation of children with violations of sound $(2,5)$.

\section{Conclusions}

The proposed technique allowed to shorten the treatment period for patients. With its application, the terms of treatment were: up to 6 months $-22.7 \%$ of people, from 6 to 12 months $-72.8 \%$, more than a year - only $4.5 \%$.

When using the standard method, $76.7 \%$ of patients used orthodontic equipment for up to 18 months.

Along with the normalization of clinical-radiological and anthropometric indicators, the application of the proposed methodology eliminated harmful habits and restored articulation motility.

The dynamics of all the studied parameters correlated with the terms of elimination of pathology and indicated the greater effectiveness of treatment in applying the proposed method, namely: all the patients normalized the form of dental arches, normalized parameters of width and length of the dental arch (in $95.4 \%$ of subjects). Immediately after the end of the treatment, electromyographic indices were normalized and occlusive relationships restored. Similar indicators for those who were treated according to the standard method, although they showed positive dynamics, but differed both from the indicators of persons who were treated according to the proposed method, and from the indicators of the control group.

Normalization of the physiological position of the tongue, both when spoken and swallowed, was slower with the increase in age of patients and the application of a common methodology. Thus, after 12 months of treatment with the application of the proposed method, the normalization of palatogram performance was observed in $95.9 \%$ of the subjects; In subgroups treated according to the standard method, similar indices were $84.1 \%$.

Thus, the developed methods of treatment will not only reduce the timing of treatment, but also normalize the impaired functions of the dental-jaw system and eliminate bad habits in patients with this pathology. 


\section{References}

1. Bezvushko E. V. Dynamics of the prevalence of dental abdominal anomalies in children of the Lviv region. Herald of problems of biology and medicine. 2015;2(2):21-24.

2. Holovko N. V. Prophylaxis of tooth-jaw abnormalities.2008;172-178.

3. Doroshenko O. M., Lykhota K. M., Doroshenko M.V., Bida O.V. Investigation of the functional state of chewing muscles in patients of different age groups with sagittal bite anomalies. Collection of scientific works of NMAPE employees n-d after P. L. Shupyk. 2015;24(2):58-63.

4. Kuroyedova V. D., Dmitrenko M. I., Makarova O. M., Stasyuk O. A. Understood orthodontics.2016:88.

5. Lykhota K. M. Comparative assessment of the effectiveness of different types of orthodontic devices in the treatment of patients with sagittal bite anomalies. Military Medicine of Ukraine.2015;4(15):34-38.

6. Lykhota K. M. Estimation of occlusive relations of the tooth-jaw system in patients with sagittal anomalies of bite by computerized occlusion. Collection of scientific works of NMAPE employees n-d after P. L. Shupyk.2015;24(3):43-50.

7. Noar Dzhozef. Practical orthodontics. Guidelines for correction of occlusion: HalDent.2015:104.

8. Petrychenko O. V. Efficiency of an integrated approach in correction of dental ankles in children with speech disorders. Problems of military health: a collection of scientific works of the Ukrainian Military Medical Academy. 2015;43:193-198.

9. Petrychenko O. V. Clinical characteristics of the maxillofacial area of orthodontic patients with speech impairment. Collection of scientific works of NMAPE employees n-d after P. L. Shupyk.2016;25:491-496.

10. Petrychenko O. V. Peculiarities of the structure of the dento-jaw area of orthodontic patients with linguistic disorders. Problems of military health: a collection of scientific works of the Ukrainian Military Medical Academy. 2017;47:222-225.

11. Flis P. S., Filonenko V. V., Doroshenko N. M. Frequency and prevalence of abnormalities and deformations of the dento-jaw machine during the period of occlusive bite. Ukrainian dental appleman.2016;1(1):75-78.

12. Shpak D. U. Features of treatment of the gnatious form of mesial bite in children in the alternating and permanent bite period.: dys.phd: 14.01.22 - Dentistry. 2016:171.

13. Graber Lee W. Orthodontics: Current Principles and Techniques. 6th Edition. 2016:928.

14. Sabrina Maniewicz Wins. Predictive factors of sagittal stability after treatment of Class II malocclusions. The Angle Orthodontist. 2016;86 (6):1033-1041. 